\title{
Stephan Krüger \\ Zur Krise der internationalen und nationalen \\ Kreditverhältnisse - Tendenzen der Kapitalakkumulation
}

\section{Von der (internationalen) Finanzkrise zur Beschäftigungskrise oder von der langfristig krisenhaften Entwicklung der Realkapitalakkumulation zur Krise des Kredits?}

Die Ende der 60er Jahre sich zeigenden Turbulenzen in den internationalen Währungsverhältnissen - zunächst sichtbar anhand der chronischen Schwäche des US-Dollar als institutionell sanktionierter Leit- und Reservewährung auf dem kapitalistischen Weltmarkt, sodann durch zweimalige Verminderung der Goldparität offiziell anerkannt, endlich durch Aufhebung der Konvertibilität des Dollars gegenüber dem Gold, d.h. durch den Zusammenbruch des internationalen Systems fixer Wechselkurse endgültig bestätigt - erscheinen rückblickend gesehen als Vorboten der spätestens seit Mitte der 70er Jahre in allen kapitalistischen Metropolen manifest gewordenen langfristig-strukturellen Krise der Kapitalakkumulation. Die historisch gesehen zweite Epoche der beschleunigten Akkumulation des Kapitals, gegründet auf eindeutige ökonomisch-politische Dominanzverhältnisse zwischen den entwickelten Nationalkapitalen auf dem Weltmarkt mit der Rolle der USA als 'Demiurgen des bürgerlichen Kosmos' (Marx) hatte ihr immanent-notwendiges Ende gefunden.

Diese Situation traf sowohl die traditionelle Nationalökonomie als auch die marxistische politische Ökonomie gänzlich unvorbereitet. Gerade der Umstand, daß sich das Ende der kapitalistischen Nachkriegsprosperität im Zerfall der seinerzeitigen Währungsverhältnisse und einer ungestümen, spekulationsgetragenen Ausdehnung der nationalen und internationalen Finanzmärkte anbahnte, also als Eklat im Geld- und Kreditsystem erschien, mobilisierte keineswegs verstärkte theoretische Anstrengungen zur fundierten Analyse. Es rächte sich für die traditionelle Ökonomie, einem kanonisierten 'Bastard-Keynesianismus' bzw. einem neoklassisch-synthetisierten 'Post-Keynesianismus' gehuldigt zu haben oder in schlichter monetaristischer Denkungsart an und für sich beliebige, empirisch konstatierte ökonomische Zusammenhänge in vermeintliche Kausalketten uminterpretiert zu haben; es zeigte sich aber auch, daß die Anfang der 70er Jahre intensiv geführte Diskussion um Probleme der Rekonstruktion der Marxschen Kritik der politischen Ökonomie in bloßen Anfängen stecken geblieben war und sich endlich in 'neo-ricardianischen' Sackgassen unauflöslich verstrickt hatte. Es bedurfte mehr als ein Jahrzehnt, bis die ökonomische Theorie den Stellenwert der Analyse von Geld und Kredit und der Finanzmärkte im Kontext eines übergreifenden Zusammenhangs 'wiederentdeckte': unter der Parole: »Zurück zum originären Keynes« wurde ein neues theoretisches Paradigma formuliert, dessen gemeinsamer Bezugspunkt - bei ansonsten vielerlei Differenzen - die Neubestimmung des gesamtwirtschaftlichen Stellenwerts der Verhältnisse der Kreditsphäre darstellt (Vgl. etwa Minsky 1975 oder auch Riese 1980 f.). Mangels fundierter theoretischer Alternativen konnte diese neue monetår-keynesianisch orientierte Richtung das Terrain der jenseits der neoklassischen Orthodoxie angesiedelten Diskussion zunächst besetzen. Mit den nötigen Einschränkungen ist dieser theoreti- 
schen Strömung auch die neuere Studie von W. Hankel (vgl. Hankel 1984) zuzurechnen; sie hat zudem den Vorzug, eine empirisch orientierte Analyse der gegenwärtigen internationalen Finanzkrise vorzugeben. Wir wollen im folgenden eine knappe, kritische Kommentierung seiner zentralen Thesen zum Ausgangspunkt nehmen.

Hankels Analyse geht von zwei zentralen Hypothesen aus. Zum einen unterstellt er ein 'unkonventionelles' Kausalitätsverbältnis zwischen finanzieller und real-reproduktiver Sphäre der Ökonomie: es sei die erstere, welche die Krise der letzteren allererst hervorbringe:

"Weltwirtschaftskrisen sind im Kern immer Finanzkrisen. Fehlt es am Geld, stockt der reale Leistungsaustausch zwischen den Staaten, gleichviel, ob Schuldnerländer fällige Zahlungen oder Überschußländer weitere Kreditierungen verweigern. Im Leistungsaustausch der Nationen läuft ohne Bezahlung nichts. Weil wir dessen ganz sicher sein können, ist auch auf eine Kausalität immer Verlaß: Blockaden des Finanztransfers verursachen Blockaden des Realtransfers, nicht umgekehrt! (Hankel 1984, S. 47). Daß Hankel mit dieser Aussage nicht den - unbestreitbaren - Sachverhalt einer krisenverschärfenden Wirkung des Kredits, nachdem die Krise bereits ausgebrochen ist, im Auge hat, erhellt aus seiner zweiten fundamentalen Hypothese: die kriseninduzierenden Blockaden der Finanztransfers resultieren aus internationalen Verwerfungen, i.e. strukturellen Zahlungsbilanzdefiziten der Nationen und wirken solcherart auf die nationalen Ökonomien nur zurück: „Es ist also das transnationale Finanzierungsklima, das das nationale bestimmt, das unkontrollierte das kontrollierte - nicht umgekehrt. Deswegen stehen die Xeno-Finanzmärkte für eine doppelte Herausforderung: Sie erhöhen die realen Krisengefahren in den nationalstaatlichen Systemen, blockieren aber gleichzeitig deren tradierte und in der Vergangenheit bewährte Antikriseninstrumente (ibid., S. 48).

In dieser Sichtweise wird die Weltwirtschaftskrise zu einem Problem der institutionellen Bedingungen der internationalen Finanzmärkte (Xeno-Märkte), die ihr Entstehen in den 60er Jahren meiner merkwürdigen Koinzidenz finanzpolitischer Illiberalität in den USA und finanzpolitischer Liberalität in den von den Kriegsschäden regenerierten westeuropäischen Ländern«(ibid., S. 52) verdanken: die Politik der Zinsbegrenzung nach oben in den USA (Regulation Q) habe zu massiven Standortverlagerungen von US-Banken zunächst nach Westeuropa geführt, weil dort höhere Zinsen zu verdienen waren bei gleichzeitig niedrigeren Refinanzierungskosten in den USA bzw. in US-Dollar. Westeuropa, d.h. London war aber nur eine Durchgangsstation der international operierenden Banken; im weiteren Verlauf verlagerten sich die Bankplätze in wesentlich aufsichtsliberalere Regionen (Vaduz, Luxemburg, Bahamas, Cayman-Islands, fernöstliche Stadtstaaten etc.). Für Hankel ist das Aufkommen der Xeno-Finanzmärkte wesentlich das Ausnützen von Abgabenprivilegien von seiten international operierender Banken, d.h. die profitable Exploitation nichtreglementierter Geschäfte von seiten des Bankkapitals. Die immer reichlicher werdende Liquidität der nationalen kapitalistischen Volkswirtschaften suchte und fand ihre internationale Verwendung über das Medium der Xeno-Finanzmärkte.

Das Prekäre dieser Kreditgeschäfte auf den Xeno-Finanzmärkten besteht Hankel zufolge aber darin, daß nicht nur der jeweilige Kreditschöpfungsmultiplikator der auf diesen Märkten operierenden Töchter der nationalen Banken wegen des Fehlens von Mindestreservevorschriften etc. per se höher ist, sondern daß zudem anstelle der Zentralbankrefinanzierung eine Refinanzierung der Xeno-Banken aus internen Bankschulden erfolgte. Der im nationalen Rahmen Sicherheit verheißende Unterschied zwischen Geld (i.e. Zentralbankgeld) und Kredit, d.h. die Regulierung der Kreditvergabepotentiale der Geschäftsbanken durch die Steuerung der Zentralbankgeldmenge, falle hier fort: der Kredit der Xeno-Banken werde seinerseits aus Kredit refinanziert, so daß im Kern eine reine Finanzwechselreiterei vorliege. 
Erst diese unkontrollierte Vergabe von Xeno-Krediten habe - so Hankel - die Finanzierung der Länderleistungsbilanzdefizite ermöglicht, mehr noch: habe diese Länderleistungsbilanzdefizite hervorgebracht, weil deflationäre Anpassungsstrategien der verschuldeten $\mathrm{Na}$ tionen - das Diktat der Zahlungsbilanz - außer Kraft gesetzt wurden. Demzufolge führe die Xeno-Markt-vermittelte Überfinanzierung allererst zur Überschuldung der Länderkunden, bis dann der rückschlagende Eklat gewaltsam die aufgebaute Kreditpyramide vernichtet:

»Wenn aber Schulden selber aus Schulden finanziert werden (müssen), tappt der dieses Geschäft besorgende private Weltbankier ohne Zentralbankrückhalt früher oder später in seine eigene Kollabierungsfalle. Dann nämlich, wenn der Weltbankier, weil seine Kunden wegen fortschreitender Überschuldung kreditunwürdig werden, seine eigene Kreditwürdigkeit verliert, sowohl gegenüber seinesgleichen: den anderen Banken des Xeno-Finanzverbundes, wie gegenüber der Gruppe echter Einleger, den OPECStazten.« (ibid,, S. 55)

Die Expansion der Xeno-Kreditmärkte und die dort vermittelte Überfinanzierung der Weltwirtschaft hat nun gewichtige Rückwirkungen auf Zinsen und Wechselkurse. Mit dem Zusammenbruch des Bretton-Woods-Währungssystems fester Wechselkurse und dem Übergang zu frei schwankenden Wechselkursen mit dem Float-Beschluß im März 1973 traten, so Hankel, an die Stelle der »regelrecht «(ibid., S. 62) zum Schutz ihrer Wechselkurse intervenierenden nationalen Zentralbanken die "regellos (ibid.) agierenden privaten Xeno-Banken:

»Mit der Entlassung der Wechselkurse in die freie Marktbewertung wurde gleichzeitig den Xeno-Finanzmärkten eine nicht gewollte Option erteilt. Denn es wurde fortan in das Belieben dieser Märkte gelegt, ob und wie stark sie bestimmte Wechselkurse hinfort leistungsbilanzgerecht auf- und niederfloaten lassen, oder ob und wie stark sie das Floating der Wechselkurse-selber durch eine kredit weise Finanzierung von Leistungsbilanzdefiziten schwacher Partner begrenzen wollten! (ibid., S. 62).

Die Grundlage für diese Fähigkeit der Xeno-Banken, die Wechselkurse zu bestimmen, liegt einfach in den quantitativen Verhältnissen, d.h. dem Umfang der Xeno-Märkte im Vergleich zu den Dispositionsmöglichkeiten der Zentralbanken. Im Resultat spiegeln die Wechselkurse kaum noch bzw. gar nicht mehr reale Kaufkraftparitäten der verschiedenen Währungen wider, sondern denaturieren zu bloßen Knappheitsprämien, die die nominale Größe ihrer Umsatzräume ausdrücken, die ihrerseits rein spekulativ bestimmt werden.

Ähnliches gilt für die Entwicklung der Zinssätze. Nachdem die weltwirtschaftliche Überfinanzierung zur Überschuldung von Länderkunden der Xeno-Finanzmärkte geführt hat, bedarf es eines progressiv ansteigenden Konsolidierungsbedarfs für kumulierte Alischulden, die aus den realen Produktionsprozessen dieser Länder nicht mehr bedient werden können. Der Z wang für die Xeno-Banken, stets neue Kredite nachzuschieben, führt zu steigenden Risikoprämien, die als Aufschlag auf die Standardzinsfüße (LIBOR-Satz) das Zinsniveau eskalieren.

"Der Prozeß der externen - und über die Xeno-Finanzmärkte importierten - Zinseskalation zerfällt (...) in zwei deutlich zu unterscheidende Phasen: In der ersten Phase steigen die Zinsen rein marktgesetzlich; es besteht eine Übernachfrage nach (zu höheren Zinsen noch erhältlichen) Konsolidierungskrediten. In der zweiten Phase steigen dagegen die Zinsen weitgehend nur noch kostenbedingt: spekulativ und risikobestimmt.« (ibid., S. 78).

Letzteres liegt wiederum in der Natur der Verfaßtheit der Xeno-Finanzmärkte: denn wer Kredite nur anbieten kann, indem er sich selbst verschuldet, kann niemals auf an und für sich uneinbringliche Forderungen verzichten. Das ganze System kann um den Preis seines Überlebens nur expandieren, niemals jedoch kontrahieren. Hierin aber liegt zugleich die in der langen Frist unvermeidliche Kollabierungsfalle. 
Damit schließt sich der Kreis der Argumentation, welcher mit der Kausalkette: Überfinanzierung - Überschuldung - Kollabierung charakterisiert werden kann und in letzter Instanz ein politisches, weil institutionelles Problem darstellt — dementsprechend auch politisch durch eine gemeinsame Anstrengung zur Entschuldung gelöst werden und für die Zukunft durch organisatorische Fortschritte im Hinblick auf die Konzeption eines neuen Weltwährungssystems mit einer 'Welt-Zentralbank' als 'lender of last ressort' an der Spitze vermieden werden kann. Aber fragen wir uns - zunächst noch ohne weitere theoretische Erörterungen -, ob dieser Analyse gefolgt werden kann?

Hinter der Summe der Xeno-Kredite in Höhe von 415 Mrd. US-Dollar in 1975 und 1610 Mrd. US-Dollar im ersten Halbjahr 1983 stünden - so Hankel - als reale Einlagen nur 37,5 bzw. 40,8 Mrd. US-Dollar der seit 1973 zum neuen Weltgläubiger avancierten OPEC-Länder. Aus dieser Relation von real verdienten Einlagen der OPEC-Länder und der geschöpften Kredite ergibt sich ein Kreditschöpfungsfaktor, der von 1:11 im Jahr 1975 auf 1:39 im ersten Halbjahr 1983 angewachsen sei:

»Von den bis 1982 aufgelaufenen Xeno-Kredit-Außenständen von 1,6 Billionen US-Dollar wurden ganze $56 \mathrm{Mrd}$ ( (=3,5\%!) durch echte OPEC-Einlagen gedeckt, $96,5 \%$ der Xeno-Kreditfinanzierungsleistung ( = rund 1,5 Billionen US-Dollar) wären somit rein kreditinflatorischer Natur gewesen: Kreditfinanzierung ohne vorangegangenen oder gleichzeitigen (realen) Sparprozeß« (ibid., S. 72).

Selbst wenn man - hierin Hankel folgend - eine überdurchschnittliche Ausleihquote vom Einlagevolumen für die Xeno-Banken aufgrund fehlender Reservevorschriften etc. unterstellt, ist diese These einer Überfinanzierung im angegebenen Ausmaß uneinsichtig. Zum einen ist es falsch, aus der Gegenüberstellung von (negativen) Leistungsbilanzsalden und (geringeren) OPEC-Überschüssen umstandslos auf eine Kreditfinanzierung der Differenz ohne realen Sparprozeß zurückzuschließen: einem negativen Saldo der Leistungsbilanz eines Landes steht ein (umgekehrter) Saldo seiner Kapitalbilanz gegenüber - daß ein langanhaltendes Leistungsbilanzdefizit durch Kapitalimporte finanziert wird, heißt noch lange nicht, daß nicht auch zur gleichen Zeit Kapitalexport, also internationale Kreditvergabe aus inländisch verdienter Ersparnis, betrieben worden ist. Hinter den vergebenen Krediten an Länder der Dritten (sowie der sog. Zweiten und Ersten) Welt, die zu einem großen Teil über die Xeno-Finanzmärkte vermittelt werden, steht daher nicht nur die Einlage der OPEC-Länder, sondern gleichermaßen das Gesamtvolumen der Kapitalexporte der kapitalistischen Metropolen, also 'reale' Sparprozesse, die nur in den jewriligen Bilanzsalden nicht mehr auftauchen. Das über die Xeno-Finanzmärkte vermittelte 'überschüssige' Geldkapital ist daher nicht, wenigstens nicht primär eine aus dem Nichts geschöpfte Überfinanzierung durch die Xeno-Banken, sondern zunächst eine 'Überersparnis' aus den kapitalistischen Akkumulationsprozessen in den Metropolen selbst. Damit relativiert sich das Bild entscheidend, denn die Koinzidenz von realen Stagnations- und Depressionstendenzen der kapitalistischen Akkumulation mit überakkumulierten, d.h. nicht reproduktiv-profitbringend anzulegendem Geldkapital ist ein so neues Phänomen nicht, sondern seit Marx, 'offiziell' spätestens seit Keynes" "General Theory « nicht nur als zufällige Koinzidenz, sondern als zwei Seiten ein und derselben Medaille wohlbekannt.

Zweitens, die Überschuldung eines Großteils der nichterdölexportierenden Entwicklungsländer, deren Bedienung aus den laufenden Exporterlösen dieser Staaten nicht mehr bewerkstelligt werden kann, besitzt seine letztendliche Grundlage in einer langfristig geschwrundenen Absorptionsfähigkeit der Reproduktionsprozesse der kapitalistischen Metropolen für industriell zu verarbeitende Rohstoffe. Ursächlich hierfür sind jedoch vollständig andere 
Gründe als die von Hankel angeführten. Der mittlerweile zu beobachtende Preisverfall für Rohstoffe auf den Weltmärkten resultiert in der zweiten Phase dann aus sekundären negativ-kumulierenden Prozessen: verengte Absatzmärkte für Produkte aus den Dritte-WeltLändern, die deren Export-Import-Relationen sukzessive verschlechtern und chronische Leistungsbilanzdefizite produzieren, daher progressive Verschuldung, provozieren ab eines gewissen Punktes verstärkte Rohstofförderung und Produktion, nur um Exportdevisen zu verdienen - bei anhaltenden und sich ihrerseits verschärfenden Nachfragerückgängen von seiten der importierenden kapitalistischen Metropolen.

Hankels Vermittlung der hier nicht bezweifelten Existenz reiner Finanztransaktionen im internationalen Maßstab mit den 'Realprozessen' innerhalb der kapitalistischen Metropolen sowie auf dem Weltmarkt ist also mangelhaft. Was er schildert, sind 'Entkoppelungsprozesse' der Geldkapitalakkumulation von der Realkapitalbildung, die als solche jedoch niemals Ausgangspunkt und Ursprung, sondern ausschließlich Konsequenzen und Resultate der bereits ausgebrochenen Krise der Kapitalakkumulation sein können. Dies heißt, daß der eigentliche Grund für die seit den70er Jahren zu konstatierende Schwäche der kapitalistischen Akkumulation nicht in den sich in der finanziellen Sphäre abspielenden Prozessen gesucht werden kann. Kehrt sich dann die zur Krise führende Kausalbeziehung zwischen Real- und Finanzsphäre um, erweist sich Hankels Verweis auf die institutionell bedingte inhärente Instabilität der Xeno-Finanzmärkte als außerordentlich wohlfeiles Argument, dem ein ganz und gar konventionelles Verständnis in bezug auf das Verhältnis zwischen 'Geld' und 'Kredit' unterliegt - als ob eine an der Kette der zentralbankkontrollierten Primärliquidität('Geld') liegende Sekundärliquidität ('Kredit') eine geldpolitisch geleitete Regulierung der finanziellen Sphäre kapitalistischer Volkswirtschaften ermöglichte! Hankel erweist sich in diesem Punkt ganz und gar als Vertreter des sog. 'Bankierstandpunktes' (Marx), dem über bankbetriebswirtschaftliche Finanzierungsregeln das Verständnis über makroökonomische Funktionszusammenhänge abhanden gekommen ist.

Beide tragenden Hypothesen der Hankelschen Argumentation teilen wir nicht, im Gegenteil. Unsere zentrale These lautet, daß sich nur über den Aufweis der widersprüchlichen Bewegungsformen der kapitalistischen (Real-) Akkumulation in der langen Frist eine Erklärung der Verselbständigung der Geldakkumulation (in allen Formen) erschließt. Die langfristig gewachsene Disproportion zwischen Realkapital- und Geldkapitalakkumulation faßt sich darüber hinaus in den internationalen Verhältnissen spezifisch zusammen und konstituiert ökonomische - im weiteren auch politische ect. - internationale Instabilitäten auf dem Weltmarkt, die ihrerseits auf die nationalen Akkumulationsprozesse von Kapital in den Metropolen - desweiteren selbstredend auch auf die Entwicklungsländer - zurückwirken und negative Multiplikatorprozesse dort selbst induzieren. Dies soll im folgenden kurz theoretisch skizziert werden.

\section{Das allgemeine Verhältnis zwischen Realkapital- und Geldkapitalakkumulation in der langen Frist}

Die langfristigen Entwicklungstendenzen der Akkumulation des Kapitals im reproduktiven Sektor ${ }^{1}$ der Volkswirtschaft sind von Marx mit dem zwiescblächtigen Gesetz des tendenziellen Falls der Durchschnittsprofitrate - Fall der Rate und absolutes Wachstum der Masse des Profits - zusammenfassend-abkürzend bezeichnet worden. Hierbei handelt es sich um nichts 
anderes als den wertmäßigen Ausdruck der prozessierenden Beziehung zwischen Produktivkraftsteigerung, Kapitalakkumulation und Kapitalentwertung in zyklenübergreifender Entwicklungsperspektive.

Zwei Einwände sind innerhalb der umfangreichen marxistischen Diskussion um dieses »in jeder Beziehung wichtigste Gesetz der modernen politischen Ökonomie» (GR, S. 634) geltend gemacht worden: zum ersten wird bezweifelt, daß die allgemeine Tendenz des Kapitals der fortwährenden Einsparung von lebendiger Arbeit und Ersatz durch Maschinerie in der langen Frist zu einer steigenden organischen oder Wertzusammensetzung des vorgeschossenen produktiven Kapitals führen müsse, zum anderen wird mit Verweis auf den Wert-Preis-Zusammenhang (sog. Transformationsproblem) die wertgesetzliche Bestimmtheit der allgemeinen oder Durchschnittsprofitrate des Gesamtkapitals in Zweifel gezogen. Okisho (vgl. Okisho 1961) hat beide fundamentalen Einwände miteinander verbunden: durch Distinktion zwischen dem 'Produktivitäts- und Kostenkriterium' - nur letzteres sei handlungsleitend und damit ökonomisch relevant - ergäbe sich im Gegensatz zu Marx' Deduktionen eine immanente Tendenz zu einer steigenden Durchschnittsprofitrate, wenn nicht steigende Reallöhne diese Bewegung konterkarieren könnten. Damit wird die Marxsche Argumentation auf den Kopf gestellt, denn Marx ging es gerade darum, zu zeigen, daß zyklisch ansteigende Geld-und Reallöhne der produktiven Arbeiter durch konzentrierte Ersatzprozesse vom fixen Kapital jeweils zu Beginn eines neuen industriellen Zyklus durch 'arbeitssparenden technischen Fortschritt' in ihrer Wirkung auf die sich zyklendurchschnittlich herausbildende allgemeine Profitrate nicht nur kompensiert, sondern sogar überkompensiert werden, so daß sich eine langfristig steigende Rate des Mehrwerts in einer sinkenden Profitrate ausdrückt. Daß dieser jeweils über die industriellen Zyklen durchgesetzte (per Saldo) 'arbeitssparende technische Fortschritt' mit einer langfristig ansteigenden organischen oder Wertzusammensetzung des Kapitals einhergeht, ist dann produktionstheoretisch auszuweisen: jede Alterierung der Preise der Produktionsfaktoren - handle es sich um sachliche Elemente des konstanten Kapitals oder - indirekt - um jeweils als notwendig anerkannte Lebensmittel für die produktiven Arbeiter (variables Kapital) - beruht, von der Gunst der Naturbedingungen abgesehen, auf einer veränderten Kombination der Produktionsfaktoren innerhalb der jeweiligen individuellen Produktionsprozesse, d.h. auf einer Einsparung lebendiger Arbeit und dem Übergang von Funktionen dieser lebendigen Arbeit auf die Maschinerie (vgl. Krüger 1985).

Die langfristig-überzyklisch beschleunigte Akkumulation des reproduktiven Gesamtkapitals, die zusammenfassend mit dem zwieschlächtigen Gesetz des tendenziellen Falls der Durchschnittsprofitrate ausgedrückt wird, bedingt jedoch nicht nur eine sukzessiv über die aufeinander folgenden industriellen Zyklen hinweg verschobene Wertstruktur des produktiv angewandten oder vorgeschossenen Kapitals, sondern zugleich eine langfristige Verschiebung des Verhältnisses zwischen produktiv fungierendem Kapital und Zirkulationskapital (i.e. Kapital in Waren- und Geldform). Wie die Entwicklungstendenzen bezüglich der verschiedenen Komponenten des produktiven Kapitalvorschusses (konstantes und variables Kapital: Wertzusammensetzung des Kapitals) ist auch die relative Abnahme des Anteils des Zirkulationskapitals am gesamten reproduktiv fungierenden Kapital, nur eine Ausdrucksform der langfristig ins Werk gesetzten Steigerung der Produktivkraft gesellschaftlicher Arbeit, die innerhalb des gesamtwirtschaftlichen Zirkulationsprozesses in Wirksamkeit tritt. Die Entwicklung des Kredit- und Bankwesens baut auf dieser Grundlage auf und potenziert durch die Kreditfunktion die beschleunigenden Wirkungen auf den Umschlag des Kapitals sowie die ökonomisierenden Prozesse. Darüber hinaus wird durch die vom Banksystem bewerkstelligte Konzentration aller zeitweilig unbeschäftigten Gelder sowohl der Umfang des gesamtwirtschaftlichen Akkumulationsfonds positiv erhöht sowie auch die jeweilige Geschwindigkeit der Kapitalakkumulation gesteigert. Allerdings bedingt diese quantitativ vermehrte und beschleunigte Akkumulation des Geldkapitals ihren spezifischen 'Preis': beides 
wird nur erreicht durch die vorgängige Verwandlung des disponiblen Geldkapitals in zinstragendes Geldkapital (Bankkapital), also durch die gleichzeitige Verwertung des bloßen Eigentumstitels auf Wert in Form des Zinses. Die durch den Kredit bewerkstelligte Beihilfe der reproduktiven Kapitalakkumulation fordert somit ihren Tribut in Gestalt der Spaltung des realisierten Bruttoprofits in Zinsen und Unternehmergewinn.

Für das reproduktive Kapital präsentiert sich die Beschleunigung und Ausweitung der Kapitalakkumulation, jeweils durchgesetzt in den Phasen der zyklischen Aufschwünge durch Inanspruchnahme von Kapitalkrediten, als langfristige Verschiebung der Kapitalstruktur zugunsten der Fremdmittel bzw. zugunsten der Außenfinanzierung. Die Eigenkapitalquote bzw. der Anteil der innerhalb des Unternehmens selbst erwirtschafteten Mittel sinkt langfristig ab; dies ist zunächst nur ein Ausdruck der langfristig über den Kredit beschleunigten Kapitalakkumulation. Jede Kreditfinanzierung von Investitionen ist daher ein zweischneidiges Schwert: einerseits wird gesamtwirtschaftlich und einzelwirtschaftlich die Größe der Akkumulationsfonds ausgedehnt und einzelwirtschaftlich die einer realen Investierung vorgelagerte Periode der sukzessiven Aufschatzung von Geldkapital bis zur Erreichung der Minimalgröße des akkumulierenden Zusatzkapitals verkürzt, andererseits wird durch den gleichen Prozeß die Position des bloßen Kapitaleigentums gegenüber dem fungierenden Kapital gestärkt.

Diese langfristige Verschiebung der Kapitalstruktur des reproduktiven Gesamtkapitals zugunsten der Fremdmittel etc. zeigt sich anhand einer langfristig steigenden Zinsquote, d.h. einer wachsenden prozentigen Belastung der realisierten Bruttoprofite durch abzuführende Vermögenseinkommen. Im Durchschnitt der jeweiligen industriellen Zyklen gesehen besitzt eine solche langfristig ansteigende Zinsquote zwei verschiedene Bestimmungsgründe: in den Aufschwungsphasen steigt der Zinsanteil am Bruttoprofit aufgrund der zunehmenden Fremdfinanzierung getätigter Erweiterungsinvestitionen, in den zyklischen Krisenphasen, in denen die Profitmasse kontrahiert, fallen Zinskosten aufgrund verstärkter Inanspruchnahme bloßer Zahlungsmittelkredite an, die zu zyklisch maximalen Zinssätzen zu bezahlen sind.

Der ansteigenden prozentigen Belastung des Bruttoprofits durch Zinsen und der so verringerten Rate des Unternehmergewinns steht auf der anderen Seite jedoch eine positiv gesteigerte Bruttoprofitmasse gegenüber. Denn es handelt sich hier nicht um die Verteilung gegebener, feststehender Größen: die Wirkung des Kredits besteht darin, daß bei je gegebenen Größen des reproduktiven Gesamtkapitals gestiegene Massen lebendiger Arbeit wert- und mehrwertproduzierend flüssig gemacht werden können. Der Kredit wirkt daher langfristig positiv auf die Akkumulationsrate des Kapitals ein. Er reproduziert jedoch nur die allgemeinen Gesetzmäßigkeiten der kapitalistischen Akkumulation; langfristig weist die Rate der Akkumulation des reproduktiven Kapitals eine fallende T'endenz, auf.

Damit ergibt sich ein zwieschlächtiges Resultat. Der Kredit verzögert und verstärkt den Fall der Nettoprofitrate oder Rate des Unternehmergewinns des reproduktiven Kapitals und er beschneidet und erhöht zugleich die langfristige Steigerungsrate der absoluten Masse dieses Nettoprofits oder Unternehmergewinns. Beide gegensätzlichen Effekte bedingen einander und bilden nur zwei Seiten ein und derselben Medaille.

Die über den Kredit bewerkstelligte quantitative Ausweitung des gesamtwirtschaftlichen Akkumulationsfonds durch Verwandlung von privater Revenueersparnis in Bankkapital und Investitionskredit konturiert auch die Antagonismen in den gesamtgesellschaftlichen Distributionsverhältnissen: das Verhältnis zwischen produktiver und individueller Kon- 
sumtion innerhalb des Reproduktionsprozesses wird zugunsten der ersteren verschoben. Dadurch wird die den gesamtwirtschaftlichen Wachstumsprozeß tragende oder stützende Rolle der konsumtiven Endnachfrage unterhöhlt: ein nur verlangsamt wachsendes langfristiges Nationaleinkommen hat einen progressiv expandierenden 'Berg' von produzierten Produktionsmitteln zu tragen. Innerhalb des Nationaleinkommens werden diejenigen Klassen und Schichten von Einkommensbeziehern durch den Bezug von Vermögenseinkommen begünstigt, die - gesamtwirtschaftlich-durchschnittlich gesehen - zu den Besserverdienenden gehören. Hierdurch wird wiederum das Niveau der Sparquote der gesamten privaten Haushalte angehoben, wodurch sich ein weiterer Rückschlag für den Gesamtumfang und die langfristige Entwicklung der individuellen Konsumtionskraft der Gesellschaft ergibt. Diesen Umstand hat Keynes im Auge, wenn er von einem in der langen Frist wachsenden Gegensatz zwischen Produktion und Konsumtion spricht. ${ }^{2}$

Soweit bisher das Verhältnis zwischen Realkapital- und Geldkapitalakkumulation in langfristig-überzyklischer Entwicklungsperspektive sich als widersprüchlich bestimmtes Verhältnis präsentierte, war dies identisch mit der Formverwandlung des reproduktiven Geldkapitals sowie der sonstigen brachliegenden Geldmittel der Gesellschaft in zinstragendes Kapital. Ihre Verwertung bestimmt sich über den Zinsfuß, d.h. hier: den Zinsfuß für langfristiges Geldkapital und seine sich über die zyklischen Schwankungen konsolidierende Bewegung in langer Frist. Abgesehen von den jeweils zyklisch-kurzfristig in der akuten Krisenphase des Zyklus sich ergebenden Verselbständigungen des kurzfristigen Geldmarkts und seines Zinsfußes gegenüber dem Kapitalmarktzinsfuß wird in der langen, zyklenübergreifenden Frist die Entwicklung des langfristigen Zinsfußes durch die realwirtschaftlich-reproduktiven Konstellationen bestimmt: langfristiges Geldkapital, welches am Geldkapitalmarkt Angebot an Leihkapital in Geldform darstellt, stammt entweder aus dem Umschlassprozeß des reproduktiven Gesamtkapitals selbst oder aus privaten Einkommen, die längerfristig gespart werden. Nachfrageseitig wird der Kapitalmarkt von der Entwicklung der Investitionskredite beherrscht, die ihrerseits der Entwicklung der reproduktiven Kapitalakkumulation in der langen Frist folgen. Hierin offenbart sich der abgeleitete Charakter des Finanzmarktes gegenüber den Märkten des reproduktiven Zirkels.

Dieses Ableitungsverhältnis drückt zugleich ein Abhängigkeitsverhältnis oder eine Hierarchie der verschiedenen Teilmärkte innerhalb der Volkswirtschaft aus. Solange die reproduktive Kapitalakkumulation langfristig in beschleunigter Weise verläuft, also nur jeweils kurzzeitig durch zyklische Rückschläge unterbrochen wird, erweist sich das reproduktive Kapital als Meister gegenüber dem zinstragenden Kapital, d.h. wird die Kapitalakkumulation durch die allgemeine Profitrate - wenn auch in kompliziert vermitteler Form - reguliert und bildet die Bewegung des Zinssatzes kein unmittelbar bestimmendes Element. Es sind die jeweiligen Profiterwartungen der Unternehmer in den zyklischen Aufschwungsphasen, welche über den Umfang der getätigten Erweiterungsinvestitionen und damit über die Entwicklung der langfristigen Akkumulationsrate des Gesamtkapitals entscheiden. Jeweils kommt es nicht auf die absolute Höhe dieser erwarteten Renditeraten auf neu anzulegendes Zusatzkapital an, sondern auf die jeweiligen Differenzen in den Grenzleistungsfähigkeiten verschiedener Investitionsalternativen. Der langfristige Zinsfuß bildet nur ein den Fächer unterschiedlicher Grenzleistungsfähigkeiten des Kapitals nach unten begrenzendes Moment: eine Realinvestition muß die Erwartung eines positiven Unternehmergewinns rechtfertigen, d.h. eine 'Verzinsung', die neben dem reinen Kapitalzins noch eine Risikoprämie etc. erbringt. Dies bedeutet, daß der Stand des Zinsfußes solange kein 
die zyklischen Investitionsprozesse behinderndes Element darstellt, solange die Grenzleistungsfähigkeit des Kapitals in den Aufschwungsphasen der Konjunkturzyklen beachtlich darüber liegt. ${ }^{3}$

Die sich über die Konjunkturzyklen hinweg konsolidierenden Bewegungen des langfristigen Durchschnittszinsfußes sind auf einer allgemeinen Ebene der Betrachtung nicht auszumachen; dies begründet sich aus dem Charakter des Zinsfußes als rein durch die Konkurrenz zwischen Leihern und Borgern bestimmter Verwertungsrate des zinstragenden Kapitals. Gleichgültig jedoch, ob der langfristige Kapitalmarktzins in der langen Frist ansteigt oder sinkt, erhält die Entwicklung des Kreditüberbaus ein wachsendes Eigenleben, welches sich in weiteren als den bisher betrachteten Wirkungen zeigt. Nicht nur nehmen die Kontributionen an das zinstragende Kapital sowohl absolut wie relativ, bezogen auf die realisierte Bruttoprofitmasse des reproduktiven Kapitals, langfristig zu; zugleich türmt sich ein langfristig wachsender 'Berg' von fiktivem Kapital auf die wertschöpfende Basis des Reproduktionsprozesses auf, der ebensoviele Belastungen derselben durch die Verwertung des bloßen Eigentumstitels auf Kapital bzw. Wert markiert: wir sprechen jetzt nicht mehr von der Akkumulation des eigentlichen (oder wirklichen) Geldkapitals, das sich bei den Banken als Depositum in verschiedener Form (money und near-money) niederschlägt, sondern von den - langfristigen - Anlageformen desselben in Wertpapieren. Jede Neuemission eines langfristigen Wertpapiers ist zunächst nichts anderes als die börsenmäßige Verkleidung einer Akquisition von Eigenkapital (bei Aktien) oder die Aufnahme von Kapitalkrediten (bei Industrieobligationen z.B.) bzw. die Aufnahme von langfristigen öffentlichen Krediten (Staatsschuldscheine). Aber jede Neuemission vermehrt den Umfang dieses fiktiven Kapitals, dessen einzelne Bestandteile eine bis zu unendliche Laufzeit (theoretisch) besitzen. Sein Preis oder Kurs stellt den zum langfristigen Zinssatz kapitalisierten festen oder variablen Ertrag dar, den der Eigentumstitel auf Wert gewährleistet. Im Maße, wie durch periodische Neuemissionen der Wert dieses fiktiven Kapitals anwächst - gesteigert oder abgeschwächt durch einen langfristig leicht fallenden oder leich $\tau$ ansteigenden Kapitalzins -, wächst das Gewicht dieses fiktiven Kapitals und wächst der Anspruch des Kapitaleigentums an die laufende Neuwertproduktion.

Eine neue Stufe der Verselbständigung dieser Geldkapitalakkumulation begründet sich jedoch nicht aus den Operationen am Emissionsmarkt, sondern am Sekundärmarkt der Börse: der Handel in umlaufenden Wertpapieren, d.h. ihr Kauf und Verkauf wirkt auf die primären Bestimmungsgründe der Zinssätze zurück und modifiziert sie im Maße, wie er langfristig an Gewicht gewinnt. Je fortgeschrittener die kapitalistische Akkumulation in einem Lande ist, je größer also die Masse der vorhandenen oder umlaufenden Effekten, desto gröBer ist dieser Sekundärmarkt der Börse und desto größer sind die Rückwirkungen der Konstellationen von Angebot und Nachfrage an der Börse auf den Geldkapitalmarkt, weil der Anteil des angebotenen bzw. nachgefragten Geldkapitals, der an der Börse zirkuliert, an der Gesamtsumme desselben wächst.

Das Prekäre dieser Rückwirkung der Börsenoperationen auf die unteren 'Stockwerke' des Kreditwesens und dadurch auf die reproduktive Kapitalakkumulation liegt nun darin, daß die Börsentransaktionen wesentlich vom spekulativen Element beherrscht werden. Was Keynes als aparten Bestandteil der gesamtwirtschaftlichen Geldmenge $M_{2}(=\mathrm{L}(\mathrm{i})$ ) gesondert berücksichtigt wissen wollte, besitzt hier seine Existenz und Wichtigkeit: es ist die jederzeitige Möglichkeit der Liquidierung einer einmal getätigten Anlage von Geldkapital für den Einzelnen - die Umschichtung seines Vermögenswert-Portfolios -, was in den Hän- 
den berufsmäßiger Spekulanten jene charakteristischen Wellen von Optimismus und Pessimismus am Effektenmarkt konstituiert, die, fernab wirklicher reproduktiver Tatbestände, sich aus sich selbst heraus alimentieren. Solange die reproduktive Kapitalakkumulation, über ihre zyklischen Schwankungen und Rückschläge hinweg, in beschleunigter Form vonstatten geht, solange wird die von Keynes so genannte 'Unternehmungslust' die 'Spekulation' (vgl. Keynes 1936, S. 133 f.) insgesamt dominieren - allerdings mit nach und nach abnehmender Kraft.

Als vorläufiges Resumée crgibt sich, daß es ein allgemeines Charakteristikum entwickelter kapitalistischer Verhältnisse ist, daß bereits auf der Grundlage einer langfristig beschleunigten Akkumulation des reproduktiven Kapitals die Akkumulation von Geldkapital in allen ihren Formen Verselbständigungstendenzen aufweist, die in einer zunehmenden Stärkung des Kapitaleigentums auf Kosten des fungierenden Kapitals und zunehmenden spekulativ induzierten - und in diesem Sinne 'autonomen' - Rückwirkungen auf Zinssatz und Geldhaltung (Liquiditätspräferenz) erscheinen. Bereits während der kapitalistischen Prosperität kommt es somit zu einer wachsenden latenten Disproportion zwischen Realkapital- und Geldkapitalakkkumulation (vgl. Krüger u. a. 1984).

Die beschleunigte Akkumulation des reproduktiven Kapitals ist die langfristige, zyklenübergreifende Bewegungsform des immanenten Widerspruchs der kapitalistischen Mehrwertproduktion. Als diese Bewegungsform ist sie ihrerseits widersprüchlich: trotz absolut wachsender Massen zyklendurchschnittlich akkumulierten (Real-)Kapitals geht die gesamtwirtschaftliche Rate der Akkumulation sukzessive zurück. Noch stärker rückläufig ist aufgrund der langfristig ansteigenden Wertzusammensetzung der Kapitalvorschüsse der langfristige Zuwachs an produktiver Beschäftigung; ebendeshalb nehmen auch die zyklendurchschnittlichen Zuwachsraten des kapitalistischen Wertprodukts langfristig ab. Dennoch gewährleistet das Zusammenspiel zwischen wachsenden Wertsummen und noch rascher expandierenden Massen stofflicher Produktionselemente (Produktivkraftentwicklung) eine gesamtwirtschaftliche beschleunigte Kapitalakkumulation. Es kommt jedoch notwendig ein Stadium, in dem das weiter wertmäßig und stofflich wachsende Kapital nur mehr eine absolut geringere produktive Arbeitsbevölkerung in Bewegung zu setzen vermag: das variable Gesamtkapital fällt nicht nur relativ im Verhältnis zum konstanten Kapital, sondern das produktivitätsinduzierte Wachstum des letzteren, d.h. die beständige Verwandlung von variablem in konstantes Kapital läßt den Umfang des variablen Kapitals selbst kontrahieren. Kann die Erhöhung des Exploitationsgrades pro Arbeitskraft den Verlust an Mehrwert- oder Profitmasse aufgrund verminderter produktiver Beschäftigung gesamtwirtschaftlich nicht mehr kompensieren, erzielt also ein wachsendes Gesamtkapital keine absolut wachsende Profitmasse mehr, erfährt die Dynamik der Realkapitalakkumulation einen strukturellen - zu unterscheiden von dem aus anderen Gründen entstehenden und stets wieder kurzzeitig 'geheilten' zyklischen - Gegenschlag: zu viel Kapital ist akkumuliert worden im Verhältnis zu den langfristig gewachsenen Verwertungsbedingungen desselben, eine Neuanlage von Kapital ist nur noch möglich bei gleichzeitiger Verdrängung bestehenden Kapitals, die beschleunigte Akkumulation des Gesamtkapitals hat einer strukturellen Überakkumulation von Kapital Platz gemacht. Der immanente Widerspruch der kapitalistischen Produktion hat sich gesamtwirtschaftlich und langfristig geltend gemacht: dem Fall der Durchschnittsprofitrate korrespondiert eine Stagnation bis Kontraktion der gesamtwirtschaftlich realisierten Profitmasse. Der Überakkumulation von Kapital entspricht auf dem anderen Pol eine wachsende Überzähligmachung von Arbeitskräften, die 
sich nach und nach nicht nur aus der fortan beschleunigt verringerten produktiven Arbeitsbevölkerung, sondern ebenso aus Lohnarbeitern nichtkapitalistischer Sphären (öffentliche Haushalte, lohnabhängige Mittelklassen) rekrutiert.

Die Erscheinungsformen dieser strukturellen Überakkumulation von Kapital unterscheiden sich zunächst nur quantitativ von denen einer 'normalen' zyklischen Krise. Allerdings lauert hinter diesem quantitativen. Unterschied eine qualitative Differenz: die Ursachen, welche die zyklische Krise bedingen und die Ursachen, welche den kapitalistischen Akkumulationsprozeß langfristig in eine strukturelle Überakkumulation von Kapital führen, sind grundverschieden. Nicht die in letzter Instanz durch zyklisch steigende Geldlöhne kurzfristig verminderten Wachstumsspielräume der entstandenen Profite des Kapitals führen zu einer unzureichend werdenden zusätzlichen Nachfrage nach Investitionsgütern, sondern die langfristigen allgemeinen Produktionsgesetze des Profits bedingen diese Erlahmung der Kapitalakkumulation, die deshalb auch durch die zyklischen 'Bereinigungsformen' der Krise nicht aufgehoben werden kann. Umgekehrt, die konsumtive Endnachfrage (privat wie öffentlich), die während des zyklischen Abschwungs als wesentlichster Stabilisator innerhalb des reproduktiven Zirkels wirkt und deren Bewegung das Ausmaß der unvermeidlichen Kapitalbrachlegungen und -entwertungen bestimmt, bevor der untere konjunkturelle Wendepunkt durchschritten werden kann, weist aufgrund der strukturellen Massenarbeitslosigkeit, die mit der langfristig gewachsenen kapitalistischen Überakkumulation einhergeht, ihrerseits mehr und mehr Erosionstendenzen auf: sie stabilisiert daher den Abschwungsprozeß nicht und hält den kontraktiven Multiplikator nicht im Zaum, sondern befördert ihn im Maße, wie historisch aufgebaute sozialstaatliche Konsumformen ihrerseits in relativ kurzer Zeit unter dem Zwang rasch steigender Defizite der gesamtwirtschaftlichen Umverteilungsfonds weggebrochen werden. Letztendlich ist daher die strukturelle Überakkumulation von Kapital identisch mit immer deutlicher hervortretenden Kontraktionsund Depressionstendenzen des gesamten ökonomischen Systems.

Ebenso wie in der zyklischen Krise bringt es die Verlangsamung des gesamtwirtschaftlichen Kapitalumschlags und die Störung des Zirkulationsprozesses an vielen Punkten seiner Peripherie mit sich, daß die strukturelle Überakkumulation von Kapital zugleich eine strukturelle Verknappung von Geldkapital darstellt, die als relative Verknappung des Angebots an Leihkapital in Geldform am Geldkapitalmarkt erscheint. Es kommt hinzu, daß die Unternehmen angesichts verengter Märkte und einer deutlich gesunkenen Auslastung ihrer produktiven Kapazitäten in erhöhtem Maße ihre Geldreservefonds aufstocken: die Vorsichtskassenhaltung steigt strukturell an und indiziert eine wachsende Liquiditätsvorliebe innerhalb des reproduktiven Sektors der Volkswirtschaft. Diese gewachsene Liquiditätsvorliebe der Unternehmer verknappt das Angebot an leihbarem Geldkapital zusätzlich, denn sie ist gleichbedeutend mit einem gestiegenen Anteil liquider Mittel, die in jeweils kurzfristigen Anlageformen gehalten werden. Relativer Rückgang des Gesamtumfangs freigesetzter Geldkapitale sowie Umschichtung ihrer Anlageformen zugunsten kurzer Festlegungszeiten markieren eine allgemeine Erscheinungsform auf seiten des Angebots am gesamtwirtschaftlichen Geldkapitalmarkt unter Bedingungen einer strukturellen Überakkumulation von Kapital.

Neben dieser (relativen) Verringerung der aus dem Kapitalumschlag herstammenden Geldkapitalien verringert sich auch die Ersparnis der Privathaushalte. Je länger die Überakkumulationskrise bereits andauert, je mehr daher auch der Umfang beschäftigungsloser Erwerbspersonen zunimmt, je mehr daher auch das Überangebot an Arbeitskräften die Be- 
schäftigten zwingt, sich Real-, später Geldeinkommenseinbußen gefallen zu lassen, um so mehr kontrahiert die laufende jährliche Gesamtersparnis der privaten Haushalte. Mehr noch: Einkommenseinbußen, die durch Rückgriff auf früher akkumulierte Reserven aufgefangen oder abgemildert werden, führen darüber hinaus zu Entsparvorgängen an vielen Punkten des gesamtwirtschaftlichen Zirkulationsprozesses; sie stützen zwar kurzfristig die Entwicklung der privaten Konsumnachfrage ab und verhindern ihre rasche Kontraktion, vermindern jedoch andererseits ebenso das Kreditvergabepotential des Bankensystems. Das gleiche gilt für die Sozialversicherungsfonds und die staatlichen Budgets.

Diese Verknappung der verschiedenen Quellen, aus denen sich das gesamtwirtschaftliche Angebot an leihbarem Geldkapital speist - ihre gemeinsame Ursache ist die Erosion der produkriven Basis des gesellschaftlichen Gesamtreproduktionsprozesses - trifft zusammen mit einer ungebrochenen Geldkapitalnachfrage am Finanzmarkt. Auf der einen Seite ist eine verlangsamte Akkumulation des reproduktiven Gesamtkapitals zwar gleichbedeutend mit einem Rückgang der Nachfrage nach Kapitalkrediten. Auf der anderen Seite markiert dieser Rückgang nach Kapitalkrediten aber keinen Rückgang der Gesamtnachfrage des reproduktiven Kapitals nach Kredit in Geldform. Wie kurzfristig in jeder zyklischen Krise wächst bei einer anhaltenden Überakkumulation von Kapital der Zahlungsmittelbedarf des Unternehmenssektors, der über den Geldkredit befriedigt werden muß, strukturell.

In dieser Nachfrage des reproduktiven Kapitals nach Zahlungsmittelkrediten zeigen sich nunmehr aber gleichzeitig signifikante Unterschiede zur bloß kurzfristig-zyklischen Krise. In jener ist es wesentlich die aus der Überproduktion von Warenkapital entspringende Inkongruenz zwischen Zahlungseingängen und durch frühere Geschäfte bedingten Zahlungsausgängen, die zu einer Anspannung der Liquiditätssituation der Unternehmen führen. Zwar mag, je nach dem Umfang der zyklischen Krise, auch der kommerzielle Kredit erschüttert sein, die volle Ausbildung des Kredit- und Bankensystems unter entwickelten kapitalistischen Verhältnissen hat jedoch dazu geführt, daß regelrechte Kreditkrisen im normalen Gang des langfristigen Akkumulationsprozesses des Kapitals weitgehend vermieden werden können. Unter Überakkumulationsbedingungen, unter denen die Zirkulationsstörungen nicht nur kurzfristiger Natur sind, sondern längerfristig-chronisch geworden sind, geht die ehemalige Stabilität des Kreditsystems mehr und mehr verloren.

Je mehr sich für die Unternehmen offenbart, daß die Verengung ihrer Absatzkanäle sich nicht mehr kurzfristig aufheben wird, wird ihre Bereitschaft zur Vergabe kommerzieller Kredite, d.h. zur Einräumung langsichtigerer Zahlungsziele gegenüber ihren industriellen und kommerziellen Abnehmern schwinden. Umfang und Laufzeit der kommerziellen Kredite gehen nach und nach strukturell zurück. In gleichem Umfang steigt der Bedarf für bare Zahlungsmittel, die bei den Banken nachgefragt werden. Es kommen aber weitere Momente hinzu: die im Zuge des ehemaligen Wachstumsprozesses zugunsten der Fremdmittel verschobene Kapitalstruktur der Unternehmen bedingt fortlaufende Tilgungs- und Zinszahlungen, die aus verringerten aktuellen Fonds erwirtschafteter Mittel bezahlt werden müssen. Wo der Überschuß (Cash Flow) der Unternehmen nick : at:sreicht, um die Verbindlichkeiten zu begleichen, entsteht neuer Kreditbedarf: alte Kredite müssen prolongiert werden, neue Kredite müssen aufgenommen werden, um Altschulden zu kegleichen. In diesem Prozeß wächst die Zinslast, die aus den erwirtschafteten Profiten beglich:n werden muß, beschleunigt an. Sie steigt absolut und relativ; sie steigt zusätzlich relativ, ${ }^{*}{ }^{*}$ reil der Umfang der realisierten Bruttoprofite kontrahiert. Das bloße Kapitaleigentum akkumuliert in dieser Situation seine Ansprüche auf zukünftig real zu produzierenden Reichtum 
beschleunigt an. Realkapitalakkumulation und Akkumulation von zinstragendem Geldkapital geraten in einen direkten Gegensatz zueinander. Die frühere, nur latente Disproportion zwischen beiden manifestiert sich im Widerspruch, da die ehemalige 'ruhige' Bewegungsform dieses innerkapitalistischen Gegensatzes weggefallen ist. Die Suprematie der Geldkapitalakkumulation, die sich nunmehr herausbildet und immer mehr verfestigt, ist jetzt der direkte Ausdruck der Schwäche der reproduktiven Kapitalbildung.

Beide hier kurz betrachteten Umstände, die relative Verknappung des Angebots an Leihkapital in Geldform sowie der strukturelle Anstieg der Kreditnachfrage des reproduktiven Sektors nach baren Zahlungsmitteln erklären schon weitgehend die charakteristische Starrheit des Zinssatzniveaus unter den Bedingungen einer strukturellen Überakkumulation von Kapital. Oberflächlich zeigt sich diese Starrheit der Zinssätze als Erhöhung ihres zyklendurchschnittlichen Niveaus. Entscheidend ist jedoch nicht so sehr diese durchschnittliche Erhöhung der Zinssätze als vielmehr ihre Rigidität nach unten, die nun zu einem weiteren Grund dafür wird, daß nach den akuten zyklischen Eklats in den konjunkturellen Abschwungsphasen die Bedingungen für einen von einer konzentrierten Ersatzbewegung des fixen Originalkapitals ausgehenden Aufschwungsprozeß verunmöglicht werden. Die strukturelle Anspannung an den Finanzmärkten tritt zu den reproduktiven Blockaden eines investitionsgetragenen Wachstumsprozesses hinzu und wirkt tendenziell auflösend auf das traditionelle Zyklusmuster.

Ein zyklischer Fall der Zinssätze nach Durchschreiten der akuten Krisis wird zudem durch weitere Bedingungen innerhalb der Kreditsphäre selbst behindert. Die chronische Liquiditätsanspannung des reproduktiven Sektors provoziert ein Verhalten der Banken, welches seinerseits im Resultat die Disproportionen zwischen reproduktivem Zirkel und Kreditsphäre verschärft. Auf der einen Seite partizipiert der Bankensektor zu einem Teil an der nunmehr etablierten Suprematie des zinstragenden Kapitals über das reproduktive Kapital. Auf der anderen Seite wird diese relative Besserstellung des Bankkapitals jedoch 'erkauft' durch eine Zunahme der Geschäftsrisiken der Banken: ein Teil ihrer Außenstände wird zweifelhaft, die Prolongation von Altkrediten durch nachgeschobene Gelder erhöht bei den Banken selbst das Illiquiditätsrisiko. Letzteres wird noch verschärft durch den Abzug von Einlagen der Sozialversicherungsfonds und der privaten Haushalte. Die Banken werden daher zum einen vorsichtiger bei der Ausreichung von Neukrediten, noch mehr bei notwendigen Umschuldungsaktionen notleidender Altkredite und beginnen zum anderen vermehrt Rücklagen zu bilden, um etwaige Forderungsausfälle zu kompensieren. D.h.: die Zuschlagssätze auf die Normalzinsen werden zunehmend großzügiger kalkuliert, die Kreditkosten also zusätzlich verteuert, die zyklischen Zinssenkungsprozesse werden nur zögerlich und in geringerem Ausmaß weitergegeben. Dieses, nach kapitalistischen Gesichtspunkten absolut rationale Geschäftsverhalten der Banken bringt es daher auch mit sich, daß die in früherer Zeit zu beobachtende Intervention der Zentralbank zugunsten eines Zinssenkungsprozesses nach überstandener zyklischer Krise immer weniger marktwirksam wird. Es ist das Verhalten der Geschäftsbanken, welches diesen Interventionsspielraum der Zentralbank zunehmend einengt und damit die Starrheit des Zinsniveaus nach unten befestigt.

Die Starrheit des Zinsniveaus nach unten verbindert nunmehr eine Neuanlage von fixem produktiven Kapital in größerem Umfang. Weiterhin hohe Kapitalzinssätze führen zu vergleichsweise gedrückten Kursen von Besitztiteln auf bereits vorhandenes Kapital. Der Kauf von Aktien, d.h. die Vornahme von Finanzinvestitionen erscheint lukrativer als die Neuanschaffung von Investitionsgütern. Im Unterschied zum zyklischen Prozeß unter den Ver- 
hältnissen einer langfristig beschleunigten Akkumulation von reproduktivem Kapital, in dem das Sinken der Zinssätze in der zweiten Phase der Abschwungsbewegung eine Wiederaufwertung des fiktiven Kapitals hervorbringt und somit eine zu zyklisch niedrigen Preisen angeschaffte neue Kapitalausrüstung wieder als rentable Anlageform von disponiblem Geldkapital erscheinen läßt, wirkt das starre Zinssatzniveau nunmehr blockierend auf die Vornahme von Realinvestierungen. Es kommt somit zu keiner konzentrierten Neuanlage von fixem Originalkapital in gesamtwirtschaftlich nennenswertem Umfang. Die Mechanismen, die in früherer Zeit den zyklischen Periodenwechsel bestimmten, sind weitgehend außer Kraft gesetzt.

Solange das gesamtwirtschaftliche Stabilisierungspotential des Reproduktionsprozesses noch eine sich aus sich selbst heraus beschleunigende Depressionsentwicklung desselben verhindert bzw. nur für einzelne Produktionszweige, nicht jedoch für die Gesamtwirtschaft zuläßt, solange also der Kredit noch in der Lage ist, Liquiditätszusammenbrüche von reproduktivem Kapital umfangsmäßig zu begrenzen und die abgeleiteten ökonomischen Sektoren des Gesamtreproduktionsprozesses, Staat und Sozialversicherung, die beschleunigte Kontraktion der gesamtgesellschaftlichen Konsumnachfrage noch aufhalten, verläuft der Akkumulationsprozeß des Kapitals noch in einer zyklischen Form. Allerdings tritt an die Stelle der früheren Aufschwungsphasen mit kumulativen, selbstverstärkenden Wachstumseffekten eine zögerliche konjunkturelle Erholung, die zudem durch unregelmäßige Oszillationsbewegungen immer wieder unterbrochen wird; an die Stelle der zyklischen Abschwünge, die in früherer Zeit zum Teil nur als Verlangsamung von Wachstumsprozessen erschienen, treten ausgeprägte, d.h. tiefgreifende und langanhaltende Kontraktionsprozesse der Gesamtproduktion. Auch innerhalb der zyklischen Erholungsphasen wirkt nunmehr jedoch das starre Zinsniveau als investitionsbegrenzendes Moment: das Messen der erwarteten Rendite disponibler Geldkapitâle âm Zinssatz auf langfristige Finanzanlagen als Kalkulationszinsfuß präjudiziert eine vermehrte Abwanderung des aus dem reproduktiven Zirkel herstammenden Geldkapitals in diefinanzielle Sphäre, dementsprechend einen deutlichen Rückgang in der gesamtwirtschaftlichen Akkumulationsquote für reproduktives Kapital.

Diese Verschiebung im Verhältnis zwischen realen Investitionsprozessen und bloß finanzieller, zinstragender Anlage von Kapital besitzt zwei miteinander zusammenhängende und aufeinander aufbauende Gründe. Zum einen ist mit dem langfristig gesunkenen Niveau der allgemeinen Profitrate die Tabelle der Grenzleistungsfähigkeit des Kapitals ebenfalls verfallen. Ihr Niveau wird jetzt zu einem entscheidenden Element, da auf der anderen Seite der gleichzeitig gestiegene bzw. nach unten starre Zinsfuß die Realrenditen übersteigt bzw. ihre positive Differenz auf ein Maß verringert, welches nicht mehr als Äquivalent unternehmerischen Risikos anzusehen ist. Es ist hierbei entscheidend, das Verhältnis der beiden Verwertungsraten von Kapital als Ausdruck langfristig gewachsener Disproportionen und Widersprüche des kapitalistischen Akkumulationsprozesses zu begreifen. Daß die Zinssätze zu hoch sind, ergibt sich aus den Konstellationen von Angebot und Nachfrage am Geldkapitalmarkt, die ihrerseits durch reproduktive Umstände bedingt sind. Daß die allgemeine Profitrate auf ein dem Zinsfuß vergleichbares Niveau gesunken ist, ist nichts anderes als das Ergebnis der vorangegangenen langfristig beschleunigten reproduktiven Kapitalakkumulation. Allerdings ist die Erscheinungsform dieser Situation an der Oberfläche eine andere: die Abhängigkeit der Zinsbewegung von den reproduktiven Verhältnissen ist verdeckt, vielmehr erscheint die gleichzeitig in stets wachsendem Umfang auf den Plan tretende Kreditnachfrage des Staates, 
zusammen mit einer restriktiven Geldpolitik der Zentralbank als zinstreibend. Auf der anderen Seite erscheint der Verfall der Rendite auf reale Investierungen nicht als immanentes Resultat ihrer widersprüchlichen Entwicklungstendenzen, sondern als Ergebnis eines strukturell gewachsenen Mißverhältnisses zwischen produktiver und unproduktiver Arbeitsverausgabung in gesamtwirtschaftlicher Dimension, die nicht der Erosion der ersteren, sondern der Expansion der letzteren zu Buche geschrieben wird. Jeweils erscheinen oberflächliche Phänomene als Gründe oder Ursachen dieser Situation und bieten solcherart die Anknüpfungspunkte für wirtschafts-etc.-politische Interventionen der öffentlichen Instanzen.

Ein auf den ersten Blick paradoxes Resultat hat sich ergeben: einerseits verknappt sich das aus dem reproduktiven Kreislauf der Ökonomie herstammende Angebot an Leihkapital in Geldform und erhöht sich der Zahlungsmittelbedarf der reproduktiven Unternehmungen zur Fortführung ihrer laufenden Reproduktion. Andererseits führt das starre und hohe Zinsniveau dazu, daß überschüssiges Kapital in Geldform entsteht und in finanzielle Anlageformen strömt. Ein Mangel an Geldkapital geht mit einem Überschuß an demselben einher. Dieses Paradoxon löst sich auf, wenn genauer betrachtet wird, für welche Kapitale einerseits dieser Mangel an Geldkapital besteht und für welche Kapitale andererseits Kassenüberschüsse sich anhäufen und zinstragend angelegt werden.

Eine wachsende Differenzierung der Verwertungs- und Liquiditätssituation einzelner Kapitale oder Kapitalgruppen ist ein notwendiges Begleitmoment der strukturellen Überakkumulation von Kapital. Diese Differenzierung schließt an die Differenz hinsichtlich des Verhältnisses zwischen Profitrate (Rendite) und Profitmasse (Gewinn) für verschieden große Kapitale an und bildet sie weiter aus. Im Unterschied zu den etablierten Unternehmungen, für die eine sinkende und niedrige Renditerate durch den absoluten Umfang der akkumulierbaren Mittel kompensiert wird, ist für kleinere und jeweils neu gebildete Kapitalanlagen die Höhe der Rate des Profits entscheidend, da sie umgekehrt die relative Geringheit der aus der Profitmasse akkumulierbaren Mittel teilweise ausgleicht und hierdurch Expansionsprozesse, die aus eigenerwirtschafteten Mitteln finanziert werden können, befördert. Mit dem langfristigen Fall der Profitrate nimmt aber die Anzahl jeweils neuanzulegender Kapitalien tendenziell ab. In diesem Sinne ist die folgende Marxsche Aussage zu verstehen:

"Die sog. Plethora des Kapitals bezieht sich immer wesentlich auf die Plethora von Kapital, für das der Fall der Profitrate nicht durch seine Masse aufgewogen wird - und dies sind immer die neu sich bildenden frischen Kapitalableger - oder auf die Plethora, welche diese, für sich selbst zur eignen Aktion unfähigen Kapitale den Leitern der großen Geschäftszweige in der Form des Kredits zur Verfügung stellt. Diese Plethora des Kapitals erwächst aus denselben Umständen, die eine relative Überbevölkerung hervorrufen, und ist daher eine diese letztre ergänzende Erscheinung, obgleich beide auf entgegengesetzten Polen stehn, unbeschäftigtes Kapital auf der einen und unbeschäftigte Arbeiterbevölkerung auf der anderen Seite« (MEW 25, S. 261)。

Nehmen wir nunmehr die Bestimmungsgründe der relativen Liquiditätspositionen einzelner Kapitalgruppen zusätzlich herein, ergibt sich ein weiterer Unterschied: gesellschaftlich organisierte Kapitale, d.h. vor allem Aktiengesellschaften verfügen im Unterschied zu Privatkapitalen über erweiterte Möglichkeiten der Außenfinanzierung über Emission neuer Aktien an der Börse. Darüber hinaus ist mit dem Sektor des gesellschaftlich organisierten Kapitals zugleich derjenige Teil der Einzelkapitale ziemlich exakt umgriffen, der im Gefolge sukzessiver Kapitalakkumulationen zu erheblichen Größenordnungen konzentriert ist. Schließlich, und hierin verbinden sich diese verschiedenen Merkmale der differentiellen Verwertungspositionen miteinander, ergibt sich aus der Organisation des Gesellschaftskapitals, daß seine norma- 
le Verwertung auch dann realisiert wird, wenn die erzielte Bruttoprofitrate unterhalb des Niveaus der durchschnittlichen Rate des gesamtwirtschaftlichen Profits liegt. Hiermit differenziert sich das Bild, welches die gesamtwirtschaftlichen Durchschnittsverhältnisse unter sich verbergen. Die im Zuge der strukturellen Überakkumulation sich mehr und mehr anspannende Liquiditätsposition des reproduktiven Gesamtkapitals betrifft aufgrund struktureller Vorteile weniger die großen Kapitale und insbesondere weniger die gesellschaftlich organisierten Unternehmungen. Der gesamtwirtschaftlich-langfristig ansteigende Verschuldungsgrad des reproduktiven Kapitals und die Auszehrung des Eigenkapitalanteils im Gefolge der strukturellen kapitalistischen Überakkumulation fällt überdurchschnittlich oder überwiegend auf die kleineren und mittleren Privatkapitale. Sie sind es, die die höchste Anteilsrate der Bankrotte auf sich vereinigen und die am härtesten von den Wellen der Zentralisationsprozesse betroffen sind. Die Last der durch die Überakkumulation von Kapital ausgelösten Verdrängungskonkurrenz zwischen den Kapitalen fällt überproportional auf diesen Sektor des privaten Eigenkapitals. Es kommt hinzu, daß aus diesen Unternehmungen zum Teil die Kapitalbasis durch Entnahmen der Eigentümer regelrecht ausgezehrt wird; diese Entnahmen bilden einen wesentlichen Teil jenes Plethora-Kapitals, welches in die Finanzanlagen strömt.

Verfolgen wir in einem letzten Schritt den Weg dieses Plethora-Kapitals noch weiter. Die Blockade der reproduktiven Kapitalakkumulation durch die hohen und nach unten starren Zinssätze gibt naturgemäß der Finanzspekulation neuen Auftrieb und hebt die spekulativen Transaktionen auf eine qualitativ neue Stufe. Erhöhte sich schon während der Phase beschleunigter reproduktiver Kapitalakkumulation mit dem Umfang der periodisch emmitierten fiktiven Kapitale die Masse der Börsentransaktionen, die nur noch sehr vermittelt oder gar nichts mehr mit reproduktiven Vorgängen zu tun hatten, so ist die Stockung und Störung der Realkapitalakkumulation nunmehr identisch mit einer neuen Blüte der Finanzspekulation. Wo die rationellen und reellen Grundlagen der von Keynes so genannten 'Unternehmungslust' aufgelöst worden sind, triumphiert die Spekulation, denn die Profitmacherei durch Überlistung der Marktpsychologie erscheint zunehmend attraktiver und erfolgversprechender als die wirkliche Profitproduktion.

Als Mittel der Finanzspekulation dient das überakkumulierte Geldkapital, welches innerhalb des reproduktiven Zirkels keine renditeträchtige Anlage mehr verspricht. Dieses Plethora-Kapital bildet jetzt nicht mehr nur zyklisch, sondern längerfristig den Teil der 'Geldmenge', den Keynes als 'Spekulationskasse' bezeichnete. Mit dem wachsenden Umfang der spekulativ verwendeten Mittel und dem steigenden Umfang der Börsentransaktionen gewinnt die durch die Spekulation generierte Eigenbewegung der Kurse des fiktiven Kapitals ein zunehmend größeres Gewicht. Es sind die tagtäglichen Schwankungen in den Kursen fiktiver Kapitale, Schwankungen, die durch die eigentümlichen Wellen von Optimismus und Pessimismus der Börse induziert werden', welche aus sich selbst heraus die Spekulation bewegen und mehr und mehr unkontrollierbarer erscheinen lassen, weil sie sich in stets größerem Maße verselbständigen gegenüber der wirklichen Ertragssituation der reproduktiven Kapitale. Ihrerseits erhöht der wachsende Umfang der Finanzspekulation den von der Börse ausgehenden rückwirkenden Einfluß der sekundären Bestimmungsgründe der Zinsbewegung gegenüber ihren primären Determinanten. Im Resultat erscheint daher tatsächlich die zukünftige Entwicklung immer weniger nach 'vernunftsgemäßen' Kalkülen antizipierbar, so daß die Wellen der 'irrationalen Massenpsychologie' an den Börsen ihre eigene Erfüllung produzieren - eine self-fullfilling prophecy. 
Damit ist der Widerspruch zwischen Realkapital- und Geldkapitalakkumulation in seinen allgemeinen Elementen bestimmt. Er erscheint als eine vollständige Trennung der Finanzsphäre von ihren realwirtschaftlich-reproduktiven Grundlagen oder als 'Entkoppelung' der Geldkapitalakkumulation von der reproduktiven Kapitalbildung. Diese Entkoppelung ist jedoch nur eine scheinbare: in Wahrheit handelt es sich um eine bereits während der ökonomischen Prosperität sich herausbildende Disproportion zwischen beiden, eine Disproportion, die eine neue Stufe der Verselbständigung erreicht, wenn die reproduktive Kapitalakkumulation durch ihre immanenten Widersprüche zersetzt wird. Diese äußerste Verselbständigung der Geldkapitalakkumulation ist der entsprechende Ausdruck, die bloße Kehrseite der strukturellen Überakkumulation von Kapital, die ihren Grund innerhalb der Produktionsbedingungen von Mehrwert und Profit besitzt. Die Bewegungen des zinstragenden Kapitals und die Entwicklungen im Kredit verschärfen die zur Überakkumulation treibenden und sie bedingenden Faktoren, sie verschärfen die Überakkumulationskrise. Dies schließt ein, daß ab eines gewissen Punktes die vom Kreditsystem ausgehenden Blockaden des kapitalistischen Akkumulationsprozesses eine Eigendynamik entfalten und schließlich weitere, eigenständige Ursachen für die strukturelle Überakkumulation von Kapital ins Leben rufen. Es zeigt dies aber nur den Charakter der Überakkumulationskrise als kapitalistischer Systemkrise.

\section{Zusammenfassung der inneren Widersprüche der Kapitalakkumulation auf dem Weltmarkt}

Die Existenz einer Periode langfristig beschleunigter Kapitalakkumulation unterstellt in der Realität einen entwickelten internationalen Konjunktur- und Akkumulationszusammenhang zwischen mehreren, auf dem kapitalistischen Weltmarkt zusammenstoßenden entwickelten Nationalkapitalen. Historisch wie begrifflich ist die Existenz des gesellschaftlichen Gesamtkapitals als Nationalkapital mit kennzeichnenden Durchschnittsverhältnissen seiner Produktions- und Verwertungsbedingungen (allgemeine Rate des Mehrwerts, durchschnittliche Wertzusammensetzung des Kapitals, durchschnittliche Umschlagsbedingungen etc.) Grundlage und Voraussetzung der Weltmarktbeziehungen des Kapitals; letztere werden mit der Etablierung des Regimes der 'großen Industrie' auf eine qualitativ neue Stufe gehoben und als Resultat der kapitalistischen Akkumulation systematisch reproduziert.

Auf den Weltmärkten bildet sich eine Stufenleiter zwischen den verschiedenen Nationalkapitalen heraus, die auf die Rangfolge der resp. Nationalarbeiten, differenziert nach Qualität, Intensität und Produktivität derselben, kurz: auf den jeweiligen Entwicklungsgrad der kapitalistischen Produktionsweise im betreffenden Land gegrïndet ist. In dieser Rangfolge der verschiedenen und unterschiedlich entwickelten Nationalkapitale ist es jeweils die am höchsten entwickelte Nation, die eine Sonderstellung einnimmt: die entwickeltste kapitalistische Nation, deren gesamtwirtschaftliche Durchschnittscharakteristika ihrer gesellschaftlichen Gesamtarbeit sie an die Spitze der universellen Arbeit setzen und deren ökonomische Überlegenheit gegenüber den anderen kapitalistischen Konkurrenten quantitativ anhand ihrer Suprematie in den Welthandelsanteilen und den internationalen Kreditverhältnissen zum Ausdruck kommt, prägt das internationale ökonomische Geschehen und übergreift die einzelnen Reproduktions- und Akkumulationsprozesse der anderen kapitalistischen Nationen. Es findet hier ein Umschlag von Resultaten in Voraussetzungen statt: die Unterschiede im Entwicklungsgrad der verschiedenen nationalen Gesamtarbeiten, die sich als Resultate des 
unterschiedlichen Entwicklungsgrads der kapitalistischen Produktionsweise in den einzelnen Ländern ergeben und in der internationalen Stufenleiter der Nationalkapitale auf dem Weltmarkt erscheinen, werden in Gestalt des jeweils entwickeltsten und daher auf dem Weltmarkt dominierenden Nationalkapitals zur Voraussetzung für die nationalen Akkumulationsprozesse des Kapitals. Die ent wickeltste kapitalist ische Nation ist nicht nur der Hegemon des internationalen Geschehens in ökonomischer und weiter politischer etc. Hinsicht, sondern - wie Marx dies treffend ausgedrückt hat - der »Deminrg des bürgerlichen Kosmos« (MEW 7, S. 440) schlechthin: Dieser Demiurg konstituiert den internationalen ökonomischen Zusammenhang als Weitmarktzyklus und in der Abfolge dieser Weltmarktzyklen die langfristigen Entwicklungstendenzen der nationalen kapitalistischen Akkumulationsprozesse.

Die Demiurgenrolle einer kapitalistischen Nation für die kapitalistische Weltwirtschaft ist qualitativ auf den dortigen entwickeltsten Stand der Produktivkraft der lebendigen Arbeit gegründet, der sich sowohl als Monopol an fortgeschrittenster Produktionstechnik oder als Monopol an bestimmten industriell erzeugten Waren zeigt und quantitativ in der Dominanz dieser Nation im Welthandel manifest wird. Gegründet auf diese Dominanz in den Handelsbeziehungen ist die Dominanz im Geldkapitalverkehr: der Weltmarktdemiurg wird zur internationalen Gläubigernation, verfügt über die größten Währungsreserven - sei dies Gold oder seine eigne nationale Währung, die zur internationalen Reserve- und Leitwährung avanciert. Gestützt auf diese internationale Hegemonieposition überträgt sich die nationale Konjunktur des Weltmarktdemiurgen über die internationale Verflechtung der Reproduktionsprozesse im Handel und der Kapitalbewegung auf die anderen kapitalistischen Metropolen, die die dominierende Konjunktur des Weltmarktdemiurgen in sekundärer oder tertiärer Form nachahmen: die Existenz des industriellen Zyklus des Weltmarktdemiurgen als Weitmarktzyklus enthält somit sowohl das Element der Konstitution der zyklischen Bewegung der nationalen Kapitalakkumulation als auch das Moment der Synchronisation der nationalen Konjunkturzyklen in sich.

Über die in den jeweiligen nationalen Konjunkturzyklen spielenden Ausgleichungsprozesse, die sich resultathaft in der Herausbildung jeweiliger nationaler Durchschnittsprofitraten für die Gesamtkapitale der Metropolen zusammenfassen, bilden sich in längerfristiger Perspektive die Entwicklungstendenzen der Kapitalakkumulation heraus: die Gesetze des internationalen Handels und weiter der internationalen Kapitalbewegung, die dem Ausgleich nationaler Zahlungsbilanzen unterliegen, bringen in längerfristigerer Entwicklungsperspektive, d.h. in der Abfolge mehrerer Konjunkturzyklen zugleich Angleichungstendenzen in den ursprïnglich verschiedenen nationalen Entwicklungsgraden der kapitalistischen Produktion und Akkumulation zustande, welche die internationale Stufenleiter der entwickelten Nationalkapitale gewissermaßen neu definieren und langfristig die ehemalige Monopolposition des Demiurgen untergraben. Diese internationalen Angleichungstendenzen, die zu einer allmählichen Verminderung der Unterschiede im Niveau der nationalen Durchschnittsprofitraten führen - Anhebung des Niveaus der Profitraten in den entwickelteren Nationen bzw. Verminderung der Progression ihres Falls, Beschleunigung der Tendenz zum Sinken der ehemals höheren nationalen Profitraten in den unterdurchschnittlich entwickelten kapitalistischen Nationen -, sind das Resultat der durch den Weltmarktzyklus modifizierten nationalen Konjunktur und insofern von den im nationalen Rahmen in und durch die zyklische Bewegung herausgesetzten Ausgleichungsprozessen der bestimmenden ökonomischen Variablen der kapitalistischen Reproduktionsprozesse zu unterscheiden. 
Diese durch nationale Ausgleichungsprozesse in mehreren aufeinander folgenden Zyklen durchgesetzten internationalen Angleichungen zwischen den verschiedenen kapitalistischen Metropolen markieren zugleich die Zusammenfassung der allgemeinen widersprüchlichen Bewegungsgesetze der Kapitalakkumulation auf dem Weltmarkt: langfristig wird die ursprünglich Stabilität gewährleistende Dominanz des Weltmarktdemiurgen unterhöhlt. Mit dem Verlust seiner führenden Position in den Welthandelsbeziehungen durch aufholende kapitalistische Konkurrenznationen entfällt auch sukzessive die realwirtschaftlich-reproduktive Basis seiner dominanten Stellung in den internationalen Finanz- und Kreditbeziehungen. Die Diskrepanz zwischen der reproduktiven Kapitalakkumulation und der international geprägten institutionell festgeschriebenen Akkumulation von Geldkapital erhält hierdurch neue Ausprägungen.

In der historischen Entwicklung der kapitalistischen Gesellschaftsformation lassen sich nach der vorindustriellen Beherrschung der internationalen Wirtschaftsbeziehungen durch das sog. 'antediluvianische' Handelskapital der norditalienischen Städterepubliken, Portugals und Hollands zwei große Epochen industrieller Entwicklung und Kapitalakkumulation unterscheiden. Datieren wir mit Marx den Beginn eines weltmarktvermittelten kapitalistischen Krisenzyklus auf das zweite Viertel des 19. Jahrhunderts (vgl. MEW 23, S. 20), so läßs sich unter dem Regime Großbritanniens als Weltmarktdemiurg die erste Periode einer beschleunigten reproduktiven Kapitalakkumulation bis zum Anfang des 20. Jahrhunderts feststellen. Bereits gegen Ende des 19. Jahrhunderts macht sich aber ein fortschreitender Abbau der Dominanzposition Großbritanniens bemerkbar. Neben dem Deutschen Reich kommen insbesondere die USA ökonomisch immer stärker auf; nur weil die USA vornehmlich für ihren inneren Markt produzieren, bleibt Großbritanien die größte Handelsnation und behält die Position des Weltgläubigers uneingeschränkt inne. Im Ergebnis des I. Weltkriegs, der die längerfristigen Umgruppierungen zwischen den kapitalistischen Metropolen beschleunigt, haben sich die weltwirtschaftlichen Gewichte weiter umverteilt. Die durch den Krieg in Europa nochmals erstarkte Position der USA reicht jedoch in der Z wischenkriegszeit nicht aus, um eine weltwirtschaftliche Neuorganisation gegen die alten kapitalistischen Mächte, insbesondere Großbritanniens, durchzusetzen. Großbritannien und Frankreich schotten sich vielmehr in ihren kolonialen Monopolen ab. Die langfristig erlahmte Dynamik der industriellen Akkumulation trifft zusammen mit einer labilen weltwirtschaftlichen Situation: wachsender Protektionismus durch Schutzzölle, Präferenzzonen und Währungsblöcke sind nur der Ausdruck eines Polyzentrismus zwischen den kapitalistischen Metropolen, in dem Großbritannien seine Position nicht mehr halten kann und die USA als neue Macht noch nicht in der Lage sind, eine (Re-) Integration des kapitalistischen Weltmarkts auf Basis des Freihandels und eines um den Dollar zentrierten Währungssytems durchzusetzen. Die Weltwirtschaftskrise $1929 \mathrm{ff}$. markiert den allfälligen Eklat, der stark durch die Desintegration des kapitalistischen Weltmarkts und labile Finanzbeziehungen zwischen den drei Zentren London, New York und Paris - sowie der nicht abzutragenden Auslandsverschuldung insbesondere des Deutschen Reiches - geprägt ist.

Rückblickend gesehen präsentiert sich diese erste Periode einer beschleunigten kapitalistischen Akkumulation als Etablierung und Vervollkommnung des Regimes der großen Industrie in den kapitalistischen Zentren. In den internationalen Beziehungen dominieren jedoch nach der kolonialen Aufteilung der Welt politisch administrative Eingriffe in die Waren-und Kapitalbeziehungen; national sind die auf der materiellen industriellen Produktion aufgebauten abgeleiteten Sektoren des Gesamtreproduktionsprozesses keineswegs zu ihrer entwickel- 
ten kapitalistischen Form gereift. Der krisenhafte Eklat am Ende der 20er Jahre ist daher zunächst und vor allem ein gewaltsamer Abbruch der beschleunigten Kapitalakkumulation, geprägt durch die international labile Situation, d.h. das Fehlen eines regulierenden weltwirtschaftlichen Zentrums und binnenwirtschaftlich verschärft durch deflationäre Austeritätspolitiken der meisten Regierungen.

Unter der Vorherrschaft der USA beginnt mit dem Ende des II. Weltkriegs die zreeite Epoche einer beschleunigten Kapitalakkumulation, die sich historisch-qualitativ von ihrem Vorläufer unterscheidet: die USA sind nun unbestritten nicht nur der größte Weltindustrieproduzent, sondern gleichermaßen die dominierende Nation auf den Weltmärkten. Erst jetzt gelingt es, die Welthandelsbeziehungen weitgehend und durch forcierten Abbau der Zollschranken etc. dem Regime des Freihandels zu subsumieren. Mit dem Bretton-Woods-Währungssystem wird die Dominanzposition der USA und ihrer Währung institutionell anerkannt und festgeschrieben. Im Zuge der beginnenden Nachkriegsprosperität werden auch innerhalb der nationalen Reproduktionsprozesse schrittweise - und in den einzelnen Ländern ungleichzeitig - die Gesamtheit der Distributions- und Umverteilungsverhältnisse ausdifferenziert; dies wirkt seinerseits stabilisierend auf die Kapitalakkumulation zurück. Die immanenten Widersprüche der kapitalistischen Akkumulation sind damit nicht verschwrunden, sie haben jedoch allererst diejenigen Bewegungsformen gefunden und erhalten, die ihre 'ruhige Ausgleichung' in der Zeit ermöglichen. Was daher vielen Zeitgenossen rück' blickend als außerordentliche - und mittlerweile wieder aufgelöste - Sonderentwicklung erscheint, ist umgekehrt allererst die adäquate Bewegung einer historisch gewordenen ökonomischen und gesellschaftlichen Totalität der kapitalistischen Produktionsweise und Gesellschafsformation auf ihrer eigenen, selbst geschaffenen und voll ausgebildeten Grundlage. ${ }^{4}$

Auf der Grundiage der nach dem II. Weltkrieg schrittweise durchgesetzten Liberalisierung des Welthandels und des Bretton-Woods-Währungssystems bilden sich in der Folgezeit neue internationale Kräfteverhältnisse heraus. Großßbritannien erlebt die Fortsetzung des Verlustes seiner internationalen Position durch die Auflösung seines Imperial Preferance System mit den Präferenzzöllen im Commonwealth und der Entkolonialisierung. Sein Weltmarktanteil sinkt kontinuierlich ab. Nach den USA bilden sich mit der Bundesrepublik und Japan zwei neue international bestimmende kapitalistische Metropolen heraus, die - obzwar Verlierernationen des II. Weltkriegs - in Europa und Südostasien unter dem Schirm der USA in der Folgezeit zu den zweit- bzw. drittmächtigsten kapitalistischen Industrie- und Handelsnationen heranwachsen. Unter dem Druck der Bildung des europäischen Marktes werden endlich auch die USA selbst zur Auflösung ihres ursprünglich protektionistischen Kurses in der Binnenwirtschaftspolitik gezwungen.

Der Abbau von Zollschranken im Welthandel wird komplettiert durch das Bretton-WoodsWährungssystem, mit dem Devisenbewirtschaftung ausgeschlossen und die freie Konvertibilität der Währungen gewährleistet werden soll. Mit den festen Wechselkursen soll fortan verhindert werden, daß Zahlungsbilanzdefizite zu Abwertungswettläufen, Importrestriktionen und Einschränkungen der Währungskonvercibilität führen; an die Stelle des Exports binnenwirtschaftlicher Schwierigkeiten mit der Tendenz der Auflösung weltmarktlicher Strukturen soll die binnenwirtschaftliche Anpassung treten: Exportstimulierung zu Lasten des Konsums mit restriktiver Flaushalts- und Geldpolitik in cen Defizitländern. An die Auflage der Zahlungsbilanzdisziplin wird auch cie Kreditvergabe seitens des Internationalen Währungsfonds geknüpf - ursprïnglich liberalere incernationale Kreditbedingungen, die auf die Er- 
richtung einer internationalen Clearing-Stelle zur Kreditvergabe ohne Auflagen an Defizitländer zielten (Keynes-Plan), wurden von den USA nicht akzeptiert. Der US-Dollar wird zur Welthandels- und reservewährung, dessen Parität zum Gold seit 1943 mit 35 Dollar pro Unze festliegt.

Damit ist die beherrschende Rolle der USA im Weltwährungssystem festgeschrieben. Sie können einmal durch ihre Dominanz im IWF die internationale Liquiditätsentwicklung steuern, sie geben zum anderen in viel größerem Umfang die Liquidität selbst durch die Entwicklung ihrer eigenen Zahlungsbilanz vor. Da der Dollar die Funktionen des Weitgelds weitgehend übernommen hat, steigt bzw. sinkt die Weltliquidität mit amerikanischen Defiziten oder Überschüssen in ihrer Zahlungsbilanz.

Im Laufe der 60er Jahre, beginnend mit dem III. Nachkriegsweltmarktzyklus (1958-1961) werden langfristige Verschiebungen im internationalen ökonomischen Gefüge sichtbar, die sich zunächst nur anhand einer beginnenden Umverteilung der Welthandelsanteile zwischen den kapitalistischen Metropolen zeigen. Sukzessive wird der Dollar aus einer überbewerteten zu einer unterbewerteten Währung. Gegen Ende der 60er Jahre, im Periodenwechsel zum V. Weltmarktzyklus, greifen zum ersten Mal spekulative Kapitalbewegungen gegen den Dollar statt. Die internationale Position der USA beginnt sich zu wandeln: ihre Schulden, die internationale Liquidität garantieren, gelten zunehmend weniger als internationale Währungsreserven denn als Schulden, als Schulden zumal, die nicht mehr völlig sicher sind. Dollars werden präsentiert, um sie in Gold einzulösen; der US-Goldvorrat, der noch 1950 das siebenfache der Dollarguthaben ausländischer Mächte betrug, schmilzt zusammen: 1967 beträgt er nur noch $78 \%$ dieser Aktiva, im Jahre 1971 war diese Quote auf $22 \%$ zurückgegangen. Verschiedene Elemente führen zu dieser Situation: neben Veränderungen in den Produktionsbedingungen des Goldes selbst, ist es vor allem ein im internationalen Vergleich sich durchsetzender Verlust der US-Wirtschaft an Konkurrenzfähigkeit, ausgedrückt in relativ verlangsamtem Produktivitätsfortschritt, der zu der Unterhöhlung der internationalen Position der USÖkonomie geführt hat. Im Mai 1971 weigert sich die Bundesbank, weiter zur Erhaltung des Dollarkurses zu intervenieren, im August 1971 erklären die USA die Goldeinlösungspflicht ihrer Währung für aufgehoben. 1973 wird das System der festen Wechselkurse endgültig zu Grabe getragen. Die ehemalige Dominanzposition der USA ist erodiert; der Zusammenbruch des Nachkriegswährungssystems ist ein bloßer Ausdruck dieser Tatsache.

Der faktische Verlust der Demiurgenrolle der USA und die erneute Herausbildung eines Polizentrismus verschiedener kapitalistischer Metropolen auf den Weltmärkten (USA, Westeuropa und Japan) ist - bei allen historischen Unterschieden - wiederum als äußere Erscheinungsform der immanenten Grenzen der beschleunigten kapiralistischen Akkumulation zu interpretieren. Vergleichbar mit der Situation in den 20er Jahren ist diese Konstellation wiederum gleichbedeutend mit einer international labilen Situation, die ihrerseits destabilisierend auf die nationalen Akkumulationsprozesse in den kapitalistischen Metropolen zurückwirkt. Anders als in der Periode der $Z$ wischenkriegszeit verhindert jedoch das historisch aufgebaute Stabilisierungspotential im internationalen wie im nationalen $\mathrm{Ma} ß s t a b$ den sofortigen katastrophischen Eklat der Weltwirtschaft.

Mit dem Zusammenbruch des Bretton Woods-Systems fester Wechselkurse und dem Übergang zum Floating setzt sich eine Bestimmung der Wechselkurse durch, die immer weniger durch realwirtschaftlich-reproduktive 'fundamentals' determiniert wird und stets mehr durch die internationale Bewegung des Geldkapitals: es ist dies der sichtbare Ausdruck der allgemein bestimmbaren Disproportion zwischen Realkapital- und Geldkapitalakkumulation 
auf dem Weltmarkt. Dies ist gleichbedeutend mit der beginnenden Dominanz spekulatv induzierter Kapitalströme und einer Einschränkung des Interventionsspielraumes der Zentralbanken; Konsequenz ist eine erhöhte Unsicherheit für die exportabhängigen Produktionen und ein zunehmender Protektionismus, der nach und nach an die Stelle des ehemaligen Freihandels-Regimes tritt. Vor diesem Hintergrund ist jede nationale Strategie zur Förderung der Exporte eines Landes gleichbedeutend mit der Verschärfung der internationalen Überakkumulationskrise, da bei dem nur noch verlangsamt wachsenden Welthandel die Verdrängung nationaler Kapitale Bedingung für die Ausweitung eigener Welthandelsanteile ist - ein weiterer Schub für protektionistische Tendenzen. Letzteres gilt sowohl im Verhältnis der kapitalistischen Metropolen zueinander als auch für die Entwicklungsländer, die gezwungen werden, ihre Auslandsverschuldung durch Exporterlöse abzuverdienen - ein angesichts der aufgelaufenen quantitativen Dimensionen der Verschuldungskrise illusorisches Unterfangen, das deflationären Tendenzen Vorschub leistet. Die unheilige Trinität von Überakkumulation, Überschuldung und Deflation, die unausweichlich zur gigantischen Depression führen muß, ist also ebenso wie in den 20 er Jahren wiederum präsent.

\section{Anmerkungen}

1 Zur terminologischen Klarstellung: mit dem von Marx geprägten Begriff des 'reproduktiven Kapitals' umschreiben wir das industrielle (und kommerzielle) Gesamtkapital einer Nation; die 'reproduktive Grundlage' der Ökonomie beinhaltet dessen Reproduktionsprozeß. Wenn wir von 'realwirtschaftlicher' oder 'Realsphäre' sprechen, meinen wir den identischen Sachverhalt - hierbei ist zu merken, daß 'Realsphäre' keine stofflichen oder Mengenverhältnisse bezeichnet, sondern genuine Wertverhältnisse.

2 Vgl. Keynes 1936, S. 26 f., 90; ebenso Krüger u.a. 1984. Entscheidend für die Bestimmung des Srellenwerts des Antagonismus in den Distributionsverhältnissen für eine Krisen- und Akkumulationstheorie ist seine Vermittlung mit den immanenten Widersprüchen in den kapitalistischen Produktionsverhältnissen selbst; dies zur Abgrenzung gegen unterkonsumtionstheoretische Krisenerklärungen.

3 Wir sprechen hier bewußt nur vom Verhältnis zwischen Grenzleistungsfähigkeit des Kapitals und Zinssatz, nicht von allgemeiner Profitrate, die als solche keine direkt handlungsbestimmende, Investitionsikalküle beeinflussende ökonomische Variable darstellt. (vgl. MEW 25, S. 378 f., sowie Bischoff/Krüger 1983) Innerhalb eines Konjunkturzyklus ist es nie die durchschnittliche Profitrate des Gesamtkapitals, deren Bewegung irgendetwas zum Verständnis kurzfristiger Prozesse beitragen würde; vielmehr sind es differenzielle Renditen, die erwartet werden für verschiedene Investitionsalternativen, desweiteren differentielle Verwertungsbedingungen für verschieden große und verschieden mit Eigenkapital (Liquidität) ausgestattete Einzelkapitale. Erst wenn im Gefolge einer längeren Periode beschleunigter Kapitalakkumulation in der Gesamtwirtschaft die gesamtwirtschaftlichen Ramenbedingungen sich verschlechtern - nachlassendes Wachstum, Behinderung des Strukturwandels (i.e. der Kapitalneuanlage und -neubildung) etc, --, Entwicklungen, die sich im tendenziellen Sinken der Durchschnittsprofitrate nur zusarnmenfassend ausdrücken, offenbart sich die krisengenerierende Wirkung einer auf ein dem Zinssatz vergleichbares Niveau gefallenen allgemeinen Profitrate. Dies aber auch nur für die Gesamtwirtschaft oder das gesamte Nationalkapital, nicht unmittelbar für einzelne Branchen und einzelne Kapitale.

4 Mit dieser kurz angeschlagenen Periodisierung der ökonomischen Entwicklung des Kapitalismus ist implizite zugleich eine Gegenposition zu den meisten, unter Rückgriff auf 'Lange-Wellen-Konzepte argumentierenden Auffassungsweisen offengelegt. 


\section{Literatur}

Bischoff/Krüger 1983: J. Bischoff/S. Krüger, Überakkumularion und industrieller Zyklus, in: J. Hoffmann (Hrsg.), Überproduktion, Unterkonsumtion, Depression, Analysen und Kontroversen zur Krisentheorie, Hamburg 1983

GR: K. Marx, Grundrisse der Kritik der politischen Ökonomie, Berlin (DFR) 1953

Hankel 1984: W. Hankel, Gegenkurs, Von der Schuldenkrise zur Vollbeschäftigung, Berlin 1984

Keynes 1936: J. M. Keynes, Allgemeine Theorie der Beschäftigung, des Zinses und des Geldes, Berlin 1936

Krüger u.a. 1984: S. Krüger u.a., Keynes kontra Marx? Darstellung und Kritik der 'General Theory', Hamburg 1984

Krüger 1985: S. Krüger, Zur allgemeinen Theorie der Kapitalakkumulation, Langfristige Entwicklung und konjunktureller Zyklus (Dissertation), erscheint voraussichtlich im Herbst 1985

MEW 7: K. Marx, F. Engels, Revue, Mai bis Oktober 1850, in: Marx-Engels-Werke Band 7, Berlin (DDR) 1972

MEW 23 u. 25: K. Marx, Das Kapital, Zur Kritik der politischen Ökonomie, I. u. III. Band, in: MEW Bde. 23 u. 25, Berlin (DDR) 1970

Minsky 1975: H.P. Minsky, John Maynard Keynes, London/Basingstoke 1975

Okishio 1961: N. Okishio, Technikal Changes and the Rate of Profit, dt: Technische Veränderungen und Profitrate, in: Nutzinger/Wolfstetter (Hrsg.), Die Marxsche Theorie und ihre Kritik, Frankfurt/New York 1974

Riese 1980 f.: H. Riese, Theorie der Geldwirtschaft, Teile A-D, unveröffentlichte Manuskripte, Berlin 1980 und 1981 